\title{
Effect on leaf photosynthetic rate by leaf blast for rice cultivars with different types and levels of resistance
}

\author{
L. Bastiaans' \& E.C. Roumen ${ }^{2}$ \\ 'Department of Theoretical Production Ecology, Wageningen Agricultural University, P.O. Box 430, \\ 6700 AK Wageningen, The Netherlands; ${ }^{2}$ Department of Plant Breeding, Wageningen Agricultural University, \\ P.O. Box 386, 6700 AJ Wageningen, The Netherlands
}

Received 11 November 1992; accepted 9 January 1993

Key words: leaf blast, Oryza sativa, photosynthesis, Pyricularia oryzae, rice, tolerance

\section{Summary}

The effect of an inoculation with Pyricularia oryzae (isolate P06-6) on net leaf photosynthetic rate of rice (Oryza sativa) was studied with four cultivars. Measurements were taken on the sixth leaf of the main culm of plants in the early tillering stage. On cultivars CO39, IR50 and IR64 a susceptible infection type developed, but a clear difference in relative infection efficiency of the cultivars was observed. The highest number of lesions developed on leaves of CO39, whereas the lowest number was found on leaves of IR64. For all three cultivars the effect of a single lesion on the reduction in net leaf photosynthetic rate was found to be equal to a reduction in leaf area of three times the area occupied by the visible lesion. On IR68, a cultivar with complete resistance, brown specks of pinpoint size appeared without any effect on net leaf photosynthetic rate.

\section{Introduction}

Leaf blast (causal organism: Pyricularia oryzae Cavara) reduces leaf photosynthetic rate of rice (Oryza sativa L.) not only through a reduction in green leaf area, but also through an effect on photosynthesis of green leaf tissue surrounding the lesion (Bastiaans, 1991). To quantify the reduction in leaf photosynthetic rate, the concept of the virtual lesion, consisting of a visual lesion and a halo, was introduced. Photosynthetic rate inside a virtual lesion is considered to be zero, and the area of the halo is chosen such that the reduction in leaf photosynthetic rate is fully accounted for.

Measurements indicated that the ratio between virtual and visual lesion size $(\beta)$ was independent of disease severity. Parameter $\beta$ is therefore a suitable measure for the effect of the pathogen on leaf pho- tosynthetic rate. Differences in $\beta$ among cultivars would imply genotypic differences in tolerance, which can be defined as the ability of the host to endure the presence of the pathogen with reduced disease symptoms and/or damage (Parlevliet, 1979). The objective of the present study was to investigate the presence of genetic variation in $\beta$. For this purpose three cultivars with a susceptible infection type, but with a different relative infection efficiency (RIE) were used. RIE is a major component of partial resistance to leaf blast in rice (Toriyama, 1975; Ahn \& Ou, 1982; Yeh \& Bonman, 1986).

The effect of inoculation with $P$. oryzae on net leaf photosynthetic rate of a cultivar with complete resistance was also determined. Smedegaard-Petersen \& Tolstrup (1985) suggested that complete resistance to a disease may have a limiting effect on yield. They referred to the energy expenses of de- 
fense reactions that inhibit or prevent pathogen growth. With incompatible powdery mildew-barley combinations they observed a temporary increase in respiration after inoculation. The second objective of this study was therefore to determine whether an inoculation with $P$. oryzae would lead to a substantial increase in respiration of a cultivar with complete resistance. Such an increase will manifest itself as a marked reduction in net leaf photosynthetic rate.

\section{Materials and methods}

\section{Plant cultivation and inoculation}

Two pot experiments were conducted in the spring of 1989, at the International Rice Research Institute (IRRI), Los Baños, the Philippines. Plants of $O$. sativa were grown in $12 \mathrm{~cm}$ diameter plastic cups. Cultivar IR50 was used as a reference. Before sowing, seeds were kept in moist Petri dishes for 5 days. Seven germinated seeds were sown per cut. Cups were randomly distributed over mobile benches of $1.5 \mathrm{~m}^{2}$. Plants were raised without standing water. Soil moisture content in the cups was monitored twice a day and water was supplied if necessary. Nitrogen fertilizer $\left(\left(\mathrm{NH}_{4}\right)_{2} \mathrm{SO}_{4}\right)$ was applied in three dressings, equivalent to $5 \mathrm{~g} \mathrm{~N} \mathrm{~m}^{-2}(0.057 \mathrm{~g} \mathrm{~N} /$ pot) each. The first dressing was applied just after emergence, the second after unfolding of the fourth leaf, and the third after unfolding of the sixth leaf.

Leaf developmental stage is known to influence both RIE and leaf photosynthetic rate, and therefore measurement of RIE and leaf photosynthetic rate were restricted to those plants on which the sixth leaf appeared three days before inoculation. Isolate P06-6 of $P$. oryzae was used for inoculation. The fungus was grown on prune agar at a temperature of $28^{\circ} \mathrm{C}$. Inoculum was prepared as described by Mackill \& Bonman (1986) and the spore density was adjusted to circa 50000 conidia ml ${ }^{-1}$. Per bench $200 \mathrm{ml}$ of inoculum was snrayed. After inoculation, the plants were incubated in a humid cage for 14 hours, at a temperature of about $25^{\circ} \mathrm{C}$.

\section{Photosynthesis measurements}

Measurements of leaf photosynthetic rate were conducted in a conditioned room with a temperature between 24 and $28^{\circ} \mathrm{C}$. Light for photosynthesis measurements was obtained by 4 HPLIT lamps (Philips, Eindhoven, The Netherlands) of $400 \mathrm{~W}$ each, mounted in a wooden frame. A waterbath, constructed of glass and filled with a water layer of $7 \mathrm{~cm}$, was installed just below the lamps to intercept heat radiation. Previous to the measurement, plants were put on a table top below the waterbath for an adaptation period of $30 \mathrm{~min}$. Leaf photosynthetic rate was determined with a portable leaf chamber analyzer (LCA; Analytical Development Co. (ADC), Hoddesdon, UK). Average conditions within the leaf chamber were: $1460 \mu \mathrm{Em}^{-2} \mathrm{~s}^{-1}$ of photosynthetically active radiation $(400-700 \mathrm{~nm}$; PAR), temperature of $32^{\circ} \mathrm{C}$, and a relative humidity of $65 \%$. The rate of photosynthesis was calculated following the procedure described by von Caemmerer \& Farquhar (1981). Leaves of the same cultivar were grouped, and specific leaf weight and nitrogen content (Kjeldahl) were determined.

\section{Experiment 1}

Plants of cultivars CO39, IR50 and IR64 were grown and inoculated. From earlier studies it was known that these cultivars develop a susceptible infection type after inoculation with isolate PO6-6, but differ in RIE (Roumen, 1992). RIE was considered as the number of sporulating lesions that developed per unit area of leaf tissue after inoculation. The presence of a gray center was used as a criterion to distinguish sporulating from non-sporulating lesions (Jeanguyot, 1983). The number of lesions was recorded six days after inoculation (DAI).

Net leaf photosynthetic rate was determined at 7 to $10 \mathrm{DAI}$. The fraction of leaf area covered with lesions (disease severity) was estimated according to the procedure described by Bastiaans (1991). Disease severity was determined for the area of leaf tissue enclosed by the leaf chamber (area A; Fig. 1). Measured leaves were classified in three categories, based on the presence and position of lesions on the 
leaf part between the leaf collar and the basal edge of the leaf chamber (area B; Fig. 1). Results within the first two categories were used to relate net photosynthetic rate of inoculated leaves $\left(P_{x}\right)$ to disease severity $(x)$, using an extended version of the model derived by Bastiaans (1991):

$$
P_{x}=\gamma P_{0}(1-x) \beta
$$

In this model, $P_{0}$ is the average leaf photosynthetic rate of comparable noninoculated leaf area. Parameters $\gamma$ and $\beta$ are proportionality factors which characterize the effect of an inoculation on leaf photosynthetic rate. For each cultivar, $\gamma$ and $\beta$ were determined by using the nonlineair least squares regression algorithm DUD (Ralston \& Jennrich, 1979) of the SAS statistical package.

\section{Experiment 2}

Plants of cultivars IR50 and IR68, a cultivar with complete resistance against isolate PO6-6, were grown and inoculated. Three DAI small brown specks of pinpoint size appeared on both cultivars. From that day onwards net leaf photosynthetic rate was determined on three consecutive days for both noninoculated and inoculated plants of IR50 and IR68. After photosynthesis measurement, total leaf area inside the leaf chamber was determined, and both the number of sporulating and non-sporulating lesions were counted.

\section{Results}

A clear difference in RIE was observed between cultivars CO39, IR50 and IR64 (Table 1). As expected, IR64 had the lowest infection frequency, whereas the infection frequency of $\mathrm{CO} 39$ was clearly the highest. Leaf nitrogen content of the three cultivars was identical.

Measurements on noninoculated plants indicated that leaf photosynthetic rate of $\mathrm{CO} 39$ was higher than that of IR50 and IR64, despite the similarity in leaf nitrogen content. In Fig. 2 the measured leaf photosynthetic rate is plotted against disease sever-

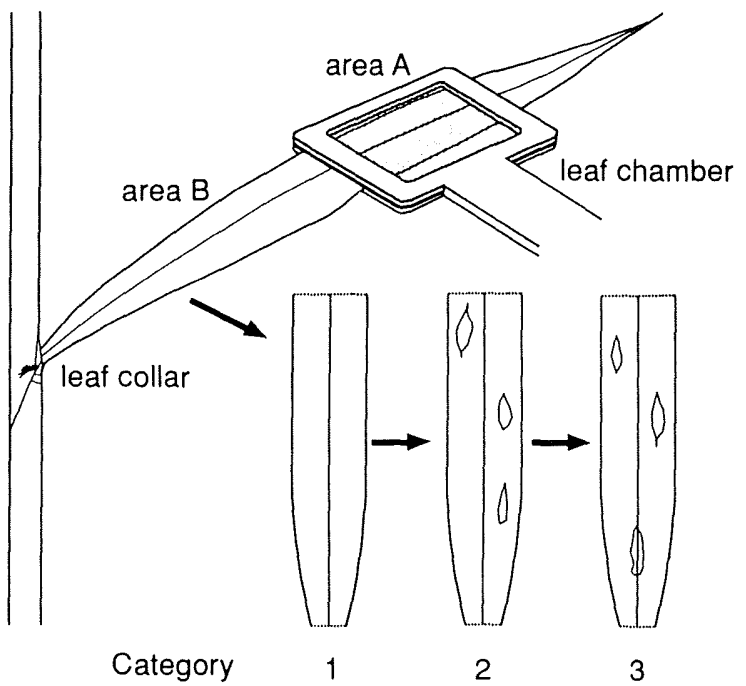

Fig. 1. Schematic representation of the measurement of net leaf photosynthetic rate. Disease severity was determined on the leaf part enclosed by the leaf chamber (area A). The presence and position of lesions between the leaf collar and the nearest edge of the leaf chamber (area B) was used to categorize measured leaves (category 1: no lesions; category 2: lesions on the laminae, but not on the central vein; category 3: lesions also on the central vein).

ity. Equation 1 described the effect of leaf blast on leaf photosynthetic rate of inoculated leaves reasonably well. Residual variance was homogeneously distributed around the fitted curves. The coefficient of variation of individual measurements of leaf photosynthetic rate (CV), calculated as the square root of the error mean square divided by the overall mean, varied between $13 \%$ (IR64) and $23 \%$ (CO39) for inoculated leaves, and between $8 \%$ (IR64) and 13\% (CO39) for noninoculated leaves. This increase in CV after inoculation can partly be explained by the experimental error in the determination of disease severity and the variation in the effect of the pathogen. Another part of the increase will be due to the imperfections of the model. Leaves of category 3 were not included in the regression. The presence of lesions on the central vein had a negative effect on the photosynthetic rate of the distal part of the leaf. On average, leaf photosynthetic rate was reduced with an extra $0.25 \mathrm{mg} \mathrm{CO}_{2} \mathrm{~m}^{-2} \mathrm{~s}^{-1}$. Such an effect was absent if lesions were only present on the laminae alongside the central vein.

The overall effect of an inoculation with $P$. oryzae 


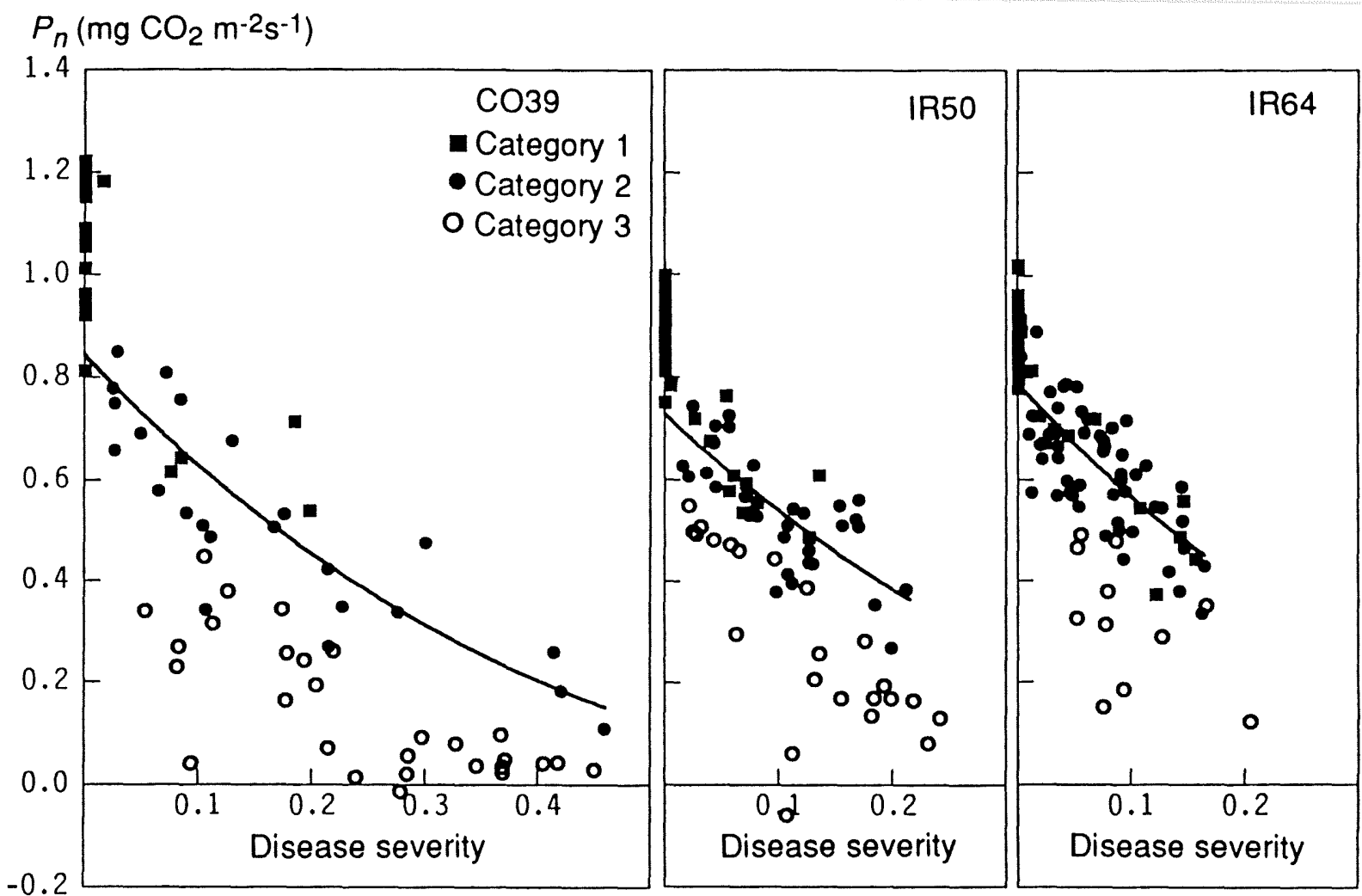

Fig. 2. Net leaf photosynthetic rate $\left(P_{n}\right)$ of three rice cultivars inoculated with Pyricularia oryzae, in relation to disease severity. Lesions were classified in three categories; see Fig. 1.

on leaf photosynthetic rate is reflected in $\gamma$ and $\beta$ (Table 1). No significant differences were found between the $\beta$-value of the three cultivars. A value of three indicates that the effect of a single lesion on leaf photosynthetic rate corresponds to a reduction in leaf area of three times the area occupied by the visible lesion. This value corresponds to earlier observations on IR50 (Bastiaans, 1991). Parameter $\gamma$

Table 1. Relative infection efficiency (RIE) and parameters characterizing the leaf photosynthetic rate $\left(P_{0}, \gamma\right.$ and $\beta:$ equation 1$)$ of three rice cultivars after inoculation with Pyricularia oryzae. Means (RIE, $\left.P_{0}\right)$ and estimated parameters $(\gamma, \beta)$ are given with their standard error. Results were obtained from leaves classified in category 1 and 2 (Fig. 1). Relevant leaf characteristics are given

\begin{tabular}{llcc}
\hline & \multicolumn{2}{l}{ Cultivar } & \\
\cline { 2 - 4 } & CO39 & IR50 & IR64 \\
\hline No. $^{a}$ & $17+28$ & $20+44$ & $26+67$ \\
RIE (lesions cm & & $1.94 \pm 0.17(\mathrm{~b})$ & $1.29 \pm 0.09(\mathrm{c})$ \\
$P_{0}\left(\mathrm{mg} \mathrm{CO}_{2} \mathrm{~m}^{-2} \mathrm{~s}^{-1}\right)$ & $3.91 \pm 0.33(\mathrm{a})^{\mathrm{b}}$ & $0.90 \pm 0.02(\mathrm{~b})$ & $0.89 \pm 0.02(\mathrm{~b})$ \\
$\gamma$ & $1.07 \pm 0.03(\mathrm{a})$ & $0.81 \pm 0.03(\mathrm{a})$ & $3.2 \pm 0.4(\mathrm{a})$ \\
$\beta$ & $0.79 \pm 0.05(\mathrm{a})$ & $2.9 \pm 0.4(\mathrm{a})$ & 1.06 \\
$\mathrm{~N}$-content $\left(\mathrm{g} \mathrm{N} \mathrm{m}^{-2}\right)$ & $2.8 \pm 0.5(\mathrm{a})$ & 1.08 & 24.6 \\
Specific leaf weight $\left(\mathrm{g} \mathrm{m}^{-2}\right)$ & 1.08 & 23.9 & $0.03(\mathrm{~b})$ \\
\hline
\end{tabular}

${ }^{a}$ Number of observations on noninoculated + inoculated leaves. ${ }^{b}$ Means in the same row, followed by the same letter are not significantly different according to t-test $(\mathrm{P}<0.05)$. 
represents the part of the effect that is not related to disease severity. It expresses the ratio between the average photosynthetic rate of inoculated but symptomless leaf area, and the photosynthetic rate of noninoculated leaf area. For all cultivars this parameter was found to be significantly smaller than one. With naturally infected leaves $\gamma$ was found to be equal to one, and therefore neglected (Bastiaans, 1991).

On cultivar IR68 small brown specks of pinpoint size were first observed on the third day after inoculation. After appearance the specks did not develop any further. This reaction is typical for incompatibility between host and pathogen. In the next days only few new specks appeared (Table 2). Simultaneously, no significant differences in photosynthetic rate were observed between leaves of noninoculated and inoculated plants of IR68.

On IR50, as on IR68, small brown specks were first observed at the third day after inoculation. The number of specks was lower than on IR68. On the fourth day after inoculation the first symptoms were observed of what later on appeared to be sporulating lesions. The number of lesions of sporulating type increased during the following days. On the day of appearance the lesions were characterized by a whitish centre surrounded by a small dark green zone. The following day the size of the whitish centre was 1.5 by $1 \mathrm{~mm}$ with a narrow brown zone around it. A significant reduction in leaf photosynthetic rate of inoculated plants of IR50 was already observed at three DAI, before the first symptoms of sporulating lesions were detected. This reduction further increased after appearance and enlargement of the lesions.

\section{Discussion}

Two parameter $(\gamma$ and $\beta$ ) were needed to characterize the effect of an inoculation with $P$. oryzae on leaf photosynthetic rate. With naturally infected leaves $\gamma$ was found to be equal to one and could therefore be neglected (Bastiaans, 1991). It is not unlikely that $\gamma$-values smaller than one are typical for artificially inoculated plants. This may be due to the development of lesions of the second generation. The first of such lesions are only expected to appear at $9 \mathrm{DAI}$ (incubation period first lesion + latent period second lesion; $5+4$ days). By that time their area hardly contributes to disease severity, but, as was demonstrated in the second experiment, leaf photosynthetic rate will already be reduced. Such a reduction is independent of disease severity, since lesions of the second generation may also originate from spores that developed on neighbouring plants.

Table 2. Net leaf photosynthetic rate $\left(P_{n} ; \mathrm{mg} \mathrm{CO}_{2} \mathrm{~m}^{-2} \mathrm{~s}^{-1}\right)$ of a susceptible (IR50) and a resistant (IR68) rice cultivar at different days after inoculation (DAI) with Pyricularia oryzae. Lesion density on inoculated leaves (lesions $\left.\mathrm{cm}^{-2}\right)$ is given for both nonsporulating $\left(\mathrm{LD}_{\text {non-sp }}\right)$ and sporulating $\left(\mathrm{LD}_{\mathrm{sp}}\right)$ lesions. Values are means \pm standard error

\begin{tabular}{|c|c|c|c|c|c|c|}
\hline \multirow{2}{*}{$\begin{array}{l}\text { IR50 } \\
\text { DAI }\end{array}$} & \multicolumn{2}{|c|}{ Noninoculated } & \multicolumn{4}{|c|}{ Inoculated } \\
\hline & No & $P_{n}$ & No & $P_{n}$ & $L D_{\text {non-sp }}$ & $\mathrm{LD}_{\mathrm{sp}}$ \\
\hline 3 & 11 & $1.03 \pm 0.03(\mathrm{a})^{\mathrm{a}}$ & 15 & $0.93 \pm 0.03(b)$ & $0.9 \pm 0.2$ & 0.0 \\
\hline 4 & 16 & $1.01 \pm 0.02(a)$ & 16 & $0.85 \pm 0.04(b)$ & $1.0 \pm 0.2$ & $1.1 \pm 0.2$ \\
\hline 5 & 14 & $1.02 \pm 0.03(a)$ & 18 & $0.79 \pm 0.04(b)$ & $1.2 \pm 0.2$ & $1.6 \pm 0.3$ \\
\hline \multirow{2}{*}{$\begin{array}{l}\text { IR68 } \\
\text { DAI }\end{array}$} & \multicolumn{2}{|c|}{ Noninoculated } & \multicolumn{4}{|c|}{ Inoculated } \\
\hline & No & $P_{n}$ & No & $P_{n}$ & $L D_{\text {non-sp }}$ & $\mathrm{LD}_{\mathrm{sp}}$ \\
\hline 3 & 13 & $1.02 \pm 0.03(\mathrm{a})$ & 15 & $1.04 \pm 0.03(\mathrm{a})$ & $1.8 \pm 0.3$ & 0.0 \\
\hline 4 & 12 & $1.01 \pm 0.03(\mathrm{a})$ & 13 & $0.99 \pm 0.04(\mathrm{a})$ & $1.8 \pm 0.5$ & 0.0 \\
\hline 5 & 15 & $1.04 \pm 0.03(a)$ & 16 & $1.01 \pm 0.02(\mathrm{a})$ & $2.2 \pm 0.5$ & 0.0 \\
\hline
\end{tabular}

${ }^{a}$ Means in the same row, followed by the same letter are not significantly different according to t-test $(\mathrm{P}<0.05)$. 
RIE (lesions $\mathrm{cm}^{-2}$ )

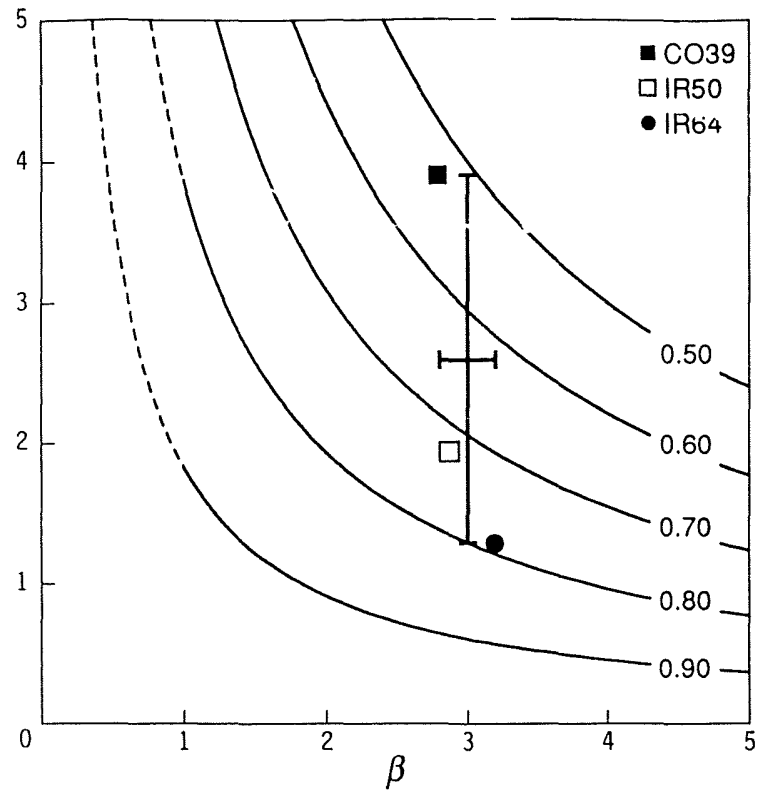

Fig. 3. Relative infection efficiency (RIE) and parameter $\beta$ as measured for three rice cultivars after inoculation with Pyricularia oryzae, and the implications for relative net leaf photosynthetic rate $\left(P_{x} P_{0}\right)$. Curves are isolines referring to combinations of RIE and $\beta$ with an equal relative net leaf photosynthetic rate (Equation 4).

Noninoculated plants however will not be affected, since they were kept separately from the inoculated plants. The significantly higher $\gamma$ of IR64 would in this case be explained by its lower RIE.

For all cultivars a $\beta$ of three was measured. This value is similar to previous results (Bastiaans, 1991). As the results do not indicate the presence of genetic variation in $\beta$, it is concluded that $\beta$ is not a suitable selection criterion in breeding rice for tolerance to leaf blast. This conclusion is emphasized in Fig. 3, where the impact of the genetic variation in $\beta$ and the impact of the genetic variation in RIE are compared. Isolines for relative leaf photosynthetic rate $\left(P_{.} / P_{0}\right)$ were constructed for combinations of $\beta$ and RIE. A function derived by Justesen \& Tammes (1960) was used to relate RIE ( $n$; lesion $\mathrm{cm}^{-2}$ ) and disease severity $(x)$ :

$$
(1-x)=(1-\alpha)^{\mathrm{n}}
$$

In this formula $\alpha$ is the area occupied by a single iesion. In the present experiment $\alpha$ was measured between 7 and 10 DAI and found to be $0.056 \mathrm{~cm}^{2}$, regardless of cultivar. This value was used in the present analysis. Substitution in equation 1 , and assuming $\gamma=1$, results in:

$$
P_{x} / P_{0}=(1-\alpha)^{n \beta}
$$

which after transformation yields the equation for the isolines:

$$
n \beta=\ln \left(P_{.} / P_{0}\right) / \ln (1-\alpha)
$$

In Fig. 3 the three cultivars are characterized by the values for $\beta$ and RIE measured in this experiment. It is shown that the differences in RIE fully account for the calculated differences in relative leaf photosynthetic rate among cultivars.

Lesions on the central vein caused a marked reduction in the photosynthetic rate of the distal part of the leaf. Disintegration of leaf-tissue in the central part of the blast lesion, as reported by Yoshii (1937), most likely resulted in a disturbed transportation of water and assimilates. Consequently, the relative water content of the leaf may be reduced, and the level of carbohydrates may be enhanced. Both effects may result in a reduction in leaf photosynthetic rate (Kaiser, 1987; Azcón-Bieto, 1983; Ku et al., 1978). Their position on the central vein enhances the impact of the referred lesions. Furthermore, these lesions were mainly found close to the leaf base were leaf width is small. This limited the possibilities of compensation through cross veinal transport. With lesions on either side of the central vein effects were localised. This observation confirms the statement of Zadoks \& Schein (1979), that lesions of equal size but in different positions do not necessarily have an equal effect on plant growth.

Incompatibility in $O$. sativa $-P$. oryzae interactions resulted in the formation of small brown specks of pinpoint size, most of which appeared at three DAI. An effect on net leaf photosynthetic rate was not observed. Also in the next two days, when only few new specks appeared, net leaf photosynthetic rate was not affected. The specks did not develop any further, and therefore an effect on three or more days after their appearance is consid- 
ered to be unlikely. A highly localized reduced net photosynthetic rate during the first days after inoculation cannot be excluded. However, the absence of a lasting effect makes the existence of a measurable effect on rice grain yield due to complete resistance against leaf blast unlikely.

\section{Acknowledgements}

The staff of the International Rice Research Institute is kindly acknowledged for co-operation and the use of their facilities. Gratitude is extended to prof. dr J.C. Zadoks and prof. dr R. Rabbinge for comments on earlier versions of the manuscript.

\section{References}

Ahn, S.W. \& S.H. Ou, 1982. Quantitative resistance of rice to blast disease. Phytopathology 72: 279-282.

Azcón-Bieto, J., 1983. Inhibition of photosynthesis by carbohydrates in wheat leaves. Plant Physiol. 73: 681-686.

Bastiaans, L., 1991. Ratio between virtual and visual lesion size as a measure to describe reduction in leaf photosynthesis of rice due to leaf blast. Phytopathology 81: 611-615.

Jeanguyot, M., 1983. Rice blast and its control. Mémoires et travaux de l'IRAT no. 3. IRAT. 53 pp.

Justesen, J.H. \& P.M.L. Tammes, 1960. Studies on yield losses. I. The self-limiting effect of injurious or competitive organisms on crop yield. Tijdschr. Plantenziekten 66: 281-287.
Kaiser, W.M., 1987. Effects of water deficit on photosynthetic capacity. Physiol. Plant. 71: 142-149.

Ku, S.B., G.E. Edwards \& D. Smith, 1978. Photosynthesis and nonstructural carbohydrate concentration in leaf blades of -Panicum virgatum as affected by night temperature. Can. J. Bot. 56: 63-68.

Mackill, A.O. \& J.M. Bonman, 1986. New Hosts of Pyricularia oryzae. Plant Dis. 70: 125-127.

Parlevliet, J.E., 1979. Components of resistance that reduce the rate of epidemic development. Ann. Rev. Phytopathol. 17: 203-222.

Ralston, M.L. \& R.I. Jennrich, 1979. DUD, a derivative-free algorthm for nonlineair least squares. Technometrics 1: 7-14.

Roumen, E.C., 1992. Effects of leaf age on components of partial resistance in rice to leaf blast. Euphytica 63: 271-279.

Smedegaard-Petersen, V. \& K. Tolstrup, 1985. The limiting effect of disease resistance on yield. Ann. Rev. Phytopathol. 23: 475490.

Toriyama, K., 1975. Recent progress of studies on horizontal resistance in rice breeding for blast resistance in Japan. In: G.E. Gàlvez (Ed.), Proc. Seminar on Horizontal Resistance to the Blast Disease of Rice, CIAT series CE-9, pp. 65-100. Cali, Colombia.

Von Caemmerer, S. \& G.D. Farquhar, 1981. Some relationships between the biochemistry of photosynthesis and the gas exchange of leaves. Planta 153: 376-387.

Yeh, W.H. \& J.M. Bonman, 1986. Assessment of partial resistance to Pyricularia oryzae in six rice cultivars. Plant Pathology 35: $319-323$

Yoshii, H., 1937. Pathological studies of rice blast caused by Pyricularia oryzae. III. Pathohistological observations of diseased plants. Ann. Phytopathol. Soc. Jpn. 7: 289-304.

Zadoks, J.C. \& R.D. Schein, 1979. Epidemiology and plant disease management. Oxford University Press, New York. 427 pp. 